\title{
Erfolgreicher Wiedereinstieg in den Beruf
}

\author{
Philippe Riedo ${ }^{a}$, Christoph Bosshard ${ }^{b}$ \\ ${ }^{a}$ Agenturleiter Suva Freiburg; ${ }^{b}$ Dr. med., Leitender Arzt Versicherungsmedizin, Suva
}

\begin{abstract}
In verschiedenen Kantonen der Schweiz entstehen Zusammenarbeitsvereinbarungen zwischen Ärzteschaft, Wirtschaftsverbänden und Sozialversicherern. Die Absicht dieses Schulterschlusses: kranke oder verunfallte Arbeitnehmende unkompliziert an den Arbeitsplatz zurückzuführen. Für Dr. med. Pierre-André Luchinger steht damit erstmals auf Papier, was er schon lange vorlebt.
\end{abstract}

Auch im Kanton Freiburg wurde vor rund zwei Jahren eine Zusammenarbeitsvereinbarung unterschrieben, initiiert von der Suva und der IV-Stelle. Sie besagt, dass alle Beteiligten bestmöglich zusammenarbeiten möchten, um kranke und verunfallte Mitarbeitende rasch und gesund in ihr gewohntes Arbeitsumfeld zurückführen zu können. «Ich hörte davon zum ersten Mal von Philippe Riedo, dem Agenturleiter der Suva Freiburg», erzählt Dr. med. Pierre-André Luchinger, Arzt für Allgemein- und Tropenmedizin mit einer Praxis in Bulle. «Ich war positiv überrascht. Als Arzt lebe ich diese Grundidee nämlich schon sehr lange. Die Zusammenarbeit ist ein wesentliches Erfolgselement für die Wiedereingliederung und eröffnet viele Möglichkeiten rund um die Genesung von kranken oder verunfallten Patientinnen und Patienten.»

\section{Der erste Schritt ist der schwierigste}

In seinen Tätigkeiten als beratender Arzt verschiedener Unternehmen fiel ihm immer wieder auf: Der Arbeitnehmer als Hauptperson wird oft nicht in diese Zusammenarbeit involviert, und alle Beteiligten erwarten, dass jemand anderes den ersten Schritt macht. Auch Ärztinnen und Ärzte haben oftmals Mühe damit, diesbezüglich die Initiative zu ergreifen. Ein Grund dafür ist das Berufsgeheimnis. «Das ist jedoch ein falscher Grund», sagt Luchinger. «Der Arbeitgeber muss keine Diagnose kennen. Er muss nur wissen, was der Mitarbeitende in welchem Ausmass machen kann, um allenfalls einen angepassten Arbeitsplatz zu finden. Häufig kennen die Ärzte aber den genauen Stellenbeschrieb nicht und können schlecht einschätzen, ob und wie lange der/die Betroffene im Moment überhaupt seiner/ihrer Tätigkeit nachgehen könnte. Sie

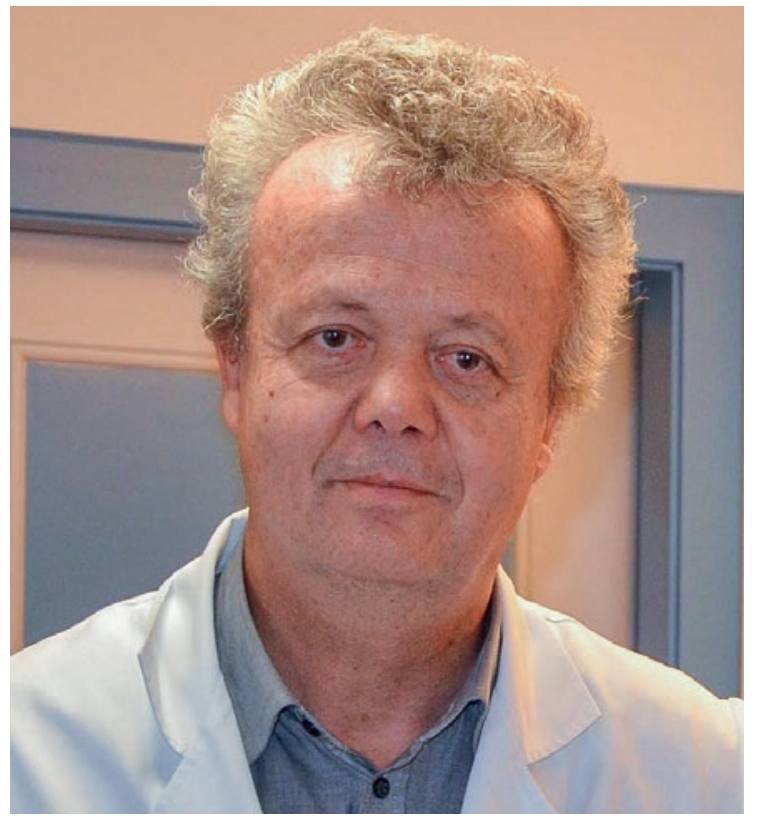

Für Pierre-André Luchinger ist klar: «Eine optimale Wiedereingliederung findet nur statt, wenn Betroffene, Führungskräfte, ärztliches Fachpersonal und Versicherungen zusammenarbeiten.»

sträuben sich vor der administrativen Papierarbeit, die eine solche Zusammenarbeit mit sich bringen könnte. Aber für all diese Hindernisse gibt es Lösungen.»

\section{Der Optimalfall}

Eigentlich müsste der Arbeitgeber die Initiative ergreifen. Nach einem Unfall müsse er gut zuhören und dem Mitarbeitenden einen Brief mitgeben, den er dem Arzt weiterreiche, skizziert Luchinger das optimale Szenario. «In diesem Brief beschreibt der Arbeitgeber, dass er eine rasche Rückkehr des Mitarbeitenden an den Ar- 
beitsplatz wünscht und bereit ist, einen angepassten Arbeitsplatz zu schaffen. Ausserdem legt er einen detaillierten Stellenbeschrieb und einen Fragebogen bei. Die Patientin oder der Patient gibt diese Unterlagen während der Konsultation beim Arzt ab, und dieser prüft sie und füllt sie aus. Das spart einerseits sehr viel Administration ein, die man direkt über die Konsultation abrechnen kann. Andererseits gewinnen alle Beteiligten viel Zeit, um eine gute Lösung zu finden. Es ist wichtig, dass solche Unterlagen nicht per Post eingeschickt werden, sondern direkt mit den Betroffenen zur Konsultation kommen. So entsteht eine persönliche, konstruktive und positive Dynamik.»

\section{Patientinnen und Patienten als Botschafter}

Die Betroffenen müssen also als Botschafterinnen und Botschafter wirken. "Das schafft eine andere Stimmung zur Mitarbeit», sagt Luchinger. «Wenn die Ärztin sieht, dass der Patient ein Interesse daran hat, baldmöglichst wieder arbeiten zu können, motiviert sie das, etwas dazu beizutragen. Deshalb sollten die Betroffenen eine proaktive Rolle einnehmen. Für sie müssen die Ärztinnen und Ärzte den administrativen Aufwand effizient halten, aber trotzdem nicht scheuen. Für sie müssen wir mit dem beratenden Arzt eines Unternehmens in Kontakt treten. Wenn die Motivation dazu von den Patientinnen und Patienten selbst kommt, bringt das am meisten, und dann fühlen sich auch die Ärztinnen motiviert. Es geht nicht darum, dass jemand mehr oder weniger Geld verdient, sondern um die Gesundheit und das Wohlergehen der Betroffenen. Daran haben alle ein Interesse.»

\section{Arbeitgebende sind genauso gefordert}

Dass Ärztinnen und Ärzte vermehrt von dieser Zusammenarbeitsvereinbarung hören und diese Idee leben, ist aus Sicht von Dr. Luchinger ein wichtiges Element. Genauso wichtig sei es aber, dass sie unter Führungskräften bekannt gemacht werde. Sie müssen einen an-

\section{Es braucht einen Arbeitgeber, der angepasste Tätigkeiten überhaupt zur Verfügung stellen kann und will.}

gepassten Arbeitsplatz anbieten können und wollen. Oft geschehe das zu spät. «Ich erlebe es viel zu häufig, dass eine Mitarbeiterin in der Firma anruft und sagt, sie habe einen Unfall erlitten und falle zwei Monate lang aus - und dann zwei Monate lang nichts mehr vom Arbeitgeber hört. Dann fühlt sie sich vergessen", sagt Luchinger.
Für grössere Unternehmen ist es häufig einfacher, angepasste Arbeitsplätze anzubieten. «Es ist aber eine Frage der Unternehmenskultur, ob ein Arbeitgeber überhaupt möchte, dass die Mitarbeitenden schnellstmöglich wieder eine Tätigkeit im Betrieb übernehmen können», sagt er. "Wer Freude an der Arbeit hat, sich mit dem Unternehmen identifiziert und mit seiner Stelle zufrieden ist, möchte rasch wieder arbeiten können. Diese Menschen brauchen einen Arbeitgeber, der angepasste Tätigkeiten überhaupt zur Verfügung stellen kann und will - auch in einem Teilzeitpensum. Es gibt zwar viele positive Beispiele, allerdings gibt es auch viele Arbeitgeber, die nur voll funktionsfähige Mitarbeitende im Betrieb wünschen.»

\section{Positive Beispiele - ob Unfall oder Krankheit}

Positive Beispiele erlebte Pierre-André Luchinger viele, und zwar sowohl nach Unfällen als auch bei Krankheitsproblemen. Ein Patient konnte mit einem externen Fixator wieder arbeiten, seine Tätigkeit wurde angepasst. Ein anderer erlitt schwerste Verbrennungen

\section{«Auch die Ärztin wird motiviert, wenn sie} sieht, dass der Patient interessiert daran ist, bald wieder zu arbeiten.»

an seinen Händen. Er war jedoch motiviert, rasch wieder zu arbeiten. Zusammen mit dem Hausarzt wurde abgeklärt, was er unter sicheren und hygienischen Bedingungen machen konnte, und er übernahm bald leichte Kontrolltätigkeiten. Ganz besonders in Erinnerung blieb ihm der Fall einer Frau, die nach 28 Jahren in der gleichen Tätigkeit entlassen wurde. Luchinger intervenierte, zeigte auf, dass man dadurch einen Sozialfall schaffe, weil die Frau nie eine andere Tätigkeit ausgeübt hatte. Das Unternehmen zog die Kündigung zurück. Auch als einem Mann gekündigt wurde, der aus psychischen Gründen Medikamente nehmen musste und diese manchmal vergass, weshalb es zu Schwierigkeiten am Arbeitsplatz kam, intervenierte Luchinger. Letztlich zog das Unternehmen auch diese Kündigung zurück - und engagierte Luchinger als beratenden Arzt für das Unternehmen.

\section{Die Krux der Arbeitsfähigkeit}

«Die schwierigste Frage ist immer, was ab wann wieder gemacht werden kann", sagt er. "Auf diese Frage kann und darf die Ärztin eine Auskunft geben, ohne auf die Verletzung oder Krankheit selbst einzugehen. Das müsste ein Arzt früh und spontan machen, was in der 
Praxis aber selten vorkommt. Häufig ist auch nicht allen bewusst, dass eine angepasste Tätigkeit nicht für den Rest des Arbeitslebens angeboten werden kann, sondern nur für den Zeitraum der Genesung. Nach drei Monaten braucht ein Unternehmen diesen Arbeitsplatz vielleicht für einen anderen verunfallten Mitarbeitenden. Hinzu kommt: Ein angepasster Arbeitsplatz setzt nicht eine hundertprozentige Arbeitsfähigkeit voraus, und ausserdem ist nicht immer allen klar, dass eine gewisse Prozentzahl der Arbeitsfähigkeit nicht unbedingt die tatsächliche Arbeitsleistung widerspiegelt. Da gibt es Klärungsbedarf.»

\section{Das Wichtigste in Kürze}

- Um kranke oder verunfallte Arbeitnehmerinnen und -nehmer unkompliziert wieder an ihre bisherige Tätigkeit heranzuführen, ist die Kooperation aller Beteiligten gefragt. Zu diesem Zwecke entstehen in verschiedenen Kantonen Zusammenarbeitsvereinbarungen zwischen Ärzten, Vertreterinnen von Unternehmen und Versicherungen.

- Dr. med. Pierre-André Luchinger aus Bulle hat auf dem Gebiet langjährige persönliche Erfahrung. Störend ist aus seiner Sicht, dass oftmals der Arbeitgeber nicht in den Wiedereingliederungsprozess involviert ist. Er empfiehlt Vorgesetzten, den Betroffenen einen Stellenbeschrieb und einen Fragebogen mitzugeben, welche diese bei der ärztlichen Konsultation abgeben. Die Ärztin oder der Arzt prüft die Unterlagen und füllt sie aus. Dies bildet die Grundlage für die Wiederaufnahme der beruflichen Tätigkeit im Rahmen des Möglichen.

\section{Zusammenarbeit ist die einzige Lösung}

«Wir haben ein starkes Versicherungssystem in der Schweiz; auch die Suva ist ein sehr erfolgreiches Modell, für das uns das Ausland beneidet», sagt Luchinger «Aber der Mensch selbst macht hier den Unterschied. Die Mitarbeitenden möchten nicht einfach ihr Gehalt am Monatsende, sie möchten einen Sinn in ihrer Arbeit erkennen, sich wohlfühlen und etwas beitragen können. Gute Führungskräfte und HR-Mitarbeitende nehmen Verantwortung wahr, haben menschliche und soziale Kompetenzen, klare Werte und investieren in Respekt und Vertrauen im Unternehmen. Das eröffnet viele Chancen. Allerdings fehlen diese Kompetenzen in vielen Unternehmen. Das ist für viel Druck und Ermüdung verantwortlich, macht die Mitarbeitenden oft passiv-aggressiv und hat einen Einfluss auf das Unfallrisiko. Ausserdem vermischen sich dann oft körperliche und psychische Beschwerden.»

Für Pierre-André Luchinger ist klar: Alle Beteiligten müssen zusammenarbeiten, für die Gesundheit der Mitarbeitenden als Patientinnen und Patienten, als Arbeitnehmende und als Versicherte. «Es gibt keine andere Lösung als diese Zusammenarbeit», sagt er. "Man muss eine positive Unternehmenskultur entwickeln und die Zusammenarbeit aller Beteiligten dynamisch und konstruktiv gestalten. Das verbessert auch unsere Gesellschaft als Ganzes.»

Bildnachweis

c MédiBulle 